\title{
POSITRON LIFETIME SPECTROSCOPY OF NANOCRYSTALLINE COPPER
}

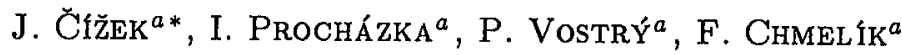 \\ AND R.K. ISLAMGALIEV ${ }^{b}$ \\ ${ }^{a}$ Faculty of Mathematics and Physics, Charles University \\ V Holešovičkách 2, 18000 Prague 8, Czech Republic \\ ${ }^{b}$ Institute of Physics of Advanced Materials \\ Ufa State Aviation Technical University \\ Ufa 450000 , Russia
}

\begin{abstract}
Positron lifetime spectroscopy was applied to investigate the thermal stability of nanocrystalline copper prepared by severe plastic torsion deformation. Positrons annihilating in as prepared specimens exhibited free positron component $\tau_{1}$ and two defect components $\tau_{2}=164$ ps and $\tau_{3}=$ 255 ps. Evolution of the lifetimes and relative intensities of all the three components with increasing annealing temperature during step-by-step isochronal annealing up to $630^{\circ} \mathrm{C}$ was studied. Behaviour of positrons in nanocrystalline copper could not be interpreted in the frame of conventional 3-state trapping model due to highly inhomogeneous defect distribution. Therefore a modified trapping model was developed and applied to explain the experimental results.
\end{abstract}

PACS numbers: $78.70 . \mathrm{Bj}, 79.60 . \mathrm{Jv}$

\section{Introduction}

Refining of grain size to submicron and nano-scale is a promising way how to improve mechanical properties of metallic materials. Nanocrystalline (NC) metals and alloys exhibit indeed, despite of other unusual properties, enhanced mechanical parameters including high strength and ductility [1]. Variety of technologies of producing NC materials have been developed so far, e.g. gas condensation method [2], rapid solidification during spark erosion [3] etc. Recently a method of processing NC metals and alloys based on severe plastic deformation (SPD) has been worked out [4].

Main feature of NC materials is significant volume fraction of grain boundaries (GB's). Previous studies [5] have shown that NC materials prepared by SPD

*Corresponding author. E-mail: jcizek@mbox.troja.mff.cuni.cz 
consist of two types of regions with different physical properties. Distorted layers with high dislocation density can be found along GB's while the regions inside grains are almost defect-free and have the same structure as in conventional coarse-grained material $[5,6]$.

Technical application of NC materials is essentially determined by thermal stability of NC structure. The present paper deals with investigation of structure evolution of nanocrystalline copper $(\mathrm{n}-\mathrm{Cu})$ with increasing annealing temperature by means of positron lifetime spectroscopy: Recent study of $n-C u$ thermal stability [7] by transmission electron microscopy (TEM), differential scanning calorimetry (DSC), electrical resistance and X-ray diffraction (XRD) clearly revealed that substantial changes of distorted regions structure connected apparently with GB dislocations precede the grain growth.

\section{Experimental details}

Positron lifetime spectrometer was similar to the fast-slow one described in [8]. The main difference was in using of summing of energy signals in slow branch. This technique is presented in [9]. Time resolution of the spectrometer was $150 \mathrm{ps}$ (FWHM for ${ }^{22} \mathrm{Na}$ ) and typical coincidence counting rate was $58 \mathrm{~s}^{-1}$. At least $13 \times 10^{6}$ counts were collected in each spectrum. Measured positron lifetime spectra (PLS) were decomposed by means of maximum-likelihood procedure [10].

Disc shaped $\mathrm{n}-\mathrm{Cu}$ specimens $(\phi 12 \mathrm{~mm} \times 0.15 \mathrm{~mm}$, purity $99.99 \%)$ were prepared by severe plastic torsion deformation as described in [4]. The samples were subjected to step-by-step isochronal annealing with effective heating rate $1^{\circ} \mathrm{C} / \mathrm{min}$ from 70 up to $630^{\circ} \mathrm{C}$. Each annealing step was finished by quenching and positron lifetime measurement at room temperature.

Application of empirical positron stopping profile [11] shows that $\approx 1 \%$ of positrons will escape through the back side of the sample if the ${ }^{22} \mathrm{Na}$ positron source $(\approx 1.3 \mathrm{MBq})$ is sandwiched between a pair of the specimens. Therefore additional identical sample was added at each side of the sandwich with purpose to prevent undesired leakage of positrons through back side of the sample and their annihilation in the air. All the four specimens were then annealed together. It was found that PLS of this multisandwich does not depend on the order of individual specimens.

\section{Results and discussion}

\subsection{Coarse-grained copper}

Coarse-grained copper ( $\phi 9 \mathrm{~mm} \times 0.5 \mathrm{~mm}$, purity $99.999 \%)$ was used as a reference specimen for determining of source components and bulk lifetime of copper. The sample was annealed at $850^{\circ} \mathrm{C}$ for 30 min. After annealing it exhibited well-recrystallized state with homogeneous coarse grains of mean linear size about $1 \mathrm{~mm}$. PLS of this sample was, except of two source contributions, purely single-component with lifetime

$$
\tau_{\mathrm{B}}=114.5 \pm 0.1[\mathrm{ps}] .
$$

This value can be attributed to the $\mathrm{Cu}$ bulk lifetime in good agreement with literature findings $[12,13]$. 


\subsection{As-prepared state of $n-C u$}

PLS of as-prepared state of $\mathrm{n}-\mathrm{Cu}$ was decomposed into three components (except of two source components). Lifetimes and relative intensitics of these components are shown in Table.

\section{TABLE}

PLS components of as-prepared state of $n-C u$.

\begin{tabular}{c|c|c|c|c|c}
\hline $\mathrm{n}-\mathrm{Cu}$ & $\tau_{1}[\mathrm{ps}]$ & $I_{1}[\%]$ & $\tau_{2}[\mathrm{ps}]$ & $I_{2}[\%]$ & $\tau_{3}[\mathrm{ps}]$ \\
\hline as-prepared & $67 \pm 8$ & $3.0 \pm 0.4$ & 164 FIX & $65 \pm 1$ & $255 \pm 2$
\end{tabular}

The shortest component $\tau_{1}$ can be obviously attributed to free positrons. Components $\tau_{2}, \tau_{3}$ originate from positrons trapped at defects in distorted regions.

Lifetime $\tau_{2}$ did not exhibit any temperature dependence during annealing (see Fig. 1a), only scattering around a mean value which is close to the Cu dislocation lifetime $\tau_{\mathrm{D}}=164 \mathrm{ps}$ [14]. Therefore all spectra were subsequently refitted with $\tau_{2}$ fixed at value $\tau_{\mathrm{D}}$ with purpose to decrease uncertainty of decomposition and to eliminate correlation between $\tau_{2}$ and other parameters. In the following discussion, only the results of this second decomposition are considered.

Migration of monovacancies was observed to start below $0^{\circ} \mathrm{C}$ in plastically deformed $\mathrm{Cu}[15]$ or around $0^{\circ} \mathrm{C}$ both in quenched [16] and electron irradiated $\mathrm{Cu}$ [17]. Doppler-broadening measurement on plastically deformed $\mathrm{Cu}$ [17] showed a marked annealing step around room temperature and did not indicate any formation of vacancy agglomerates. It is generally accepted that higher density of defects decreases their effective migration energy which implies a lower temperature of recovery. In our case the high dislocation density in distorted regions increases number of possible sinks for monovacancies. It means that the migration stage of monovacancies may be shifted even to lower temperatures for the present specimen. Therefore the component $\tau_{2}$ is assumed to be a contribution of dislocations and no contribution of single monovacancies is considered. This assumption is supported by high thermal stability of this component (Fig. Ib) and no temperature dependence of $\tau_{2}$ (Fig. 1a).

TEM studies of $\mathrm{n}-\mathrm{Cu}$ [7] revealed high dislocation density in distorted regions. Moreover, XRD investigations [6, 7] showed strongly non-uniform distribution of dislocations. Therefore we assume that the component $\tau_{2}$ comes from positrons trapped by dislocations in distorted regions.

The third component with lifetime $\tau_{3}$ is connected with larger defects whose free volume roughly corresponds to that of about 5 monovacancies [17]. In previous positron lifetime investigations of NC metals prepared both by GCM [18] and SPD [19] the authors denoted these defects as "nanovoids". "Nanovoids" are expected to be situated at intersections of GB's ("triple points"). Because of their high thermal stability (Fig. 1b) "nanovoids" seem to be some structure elements of NC structure. Recently similar thermal stability of "nanovoids" was found also in $\mathrm{n}-\mathrm{Cu}$ prepared by GCM [18]. 

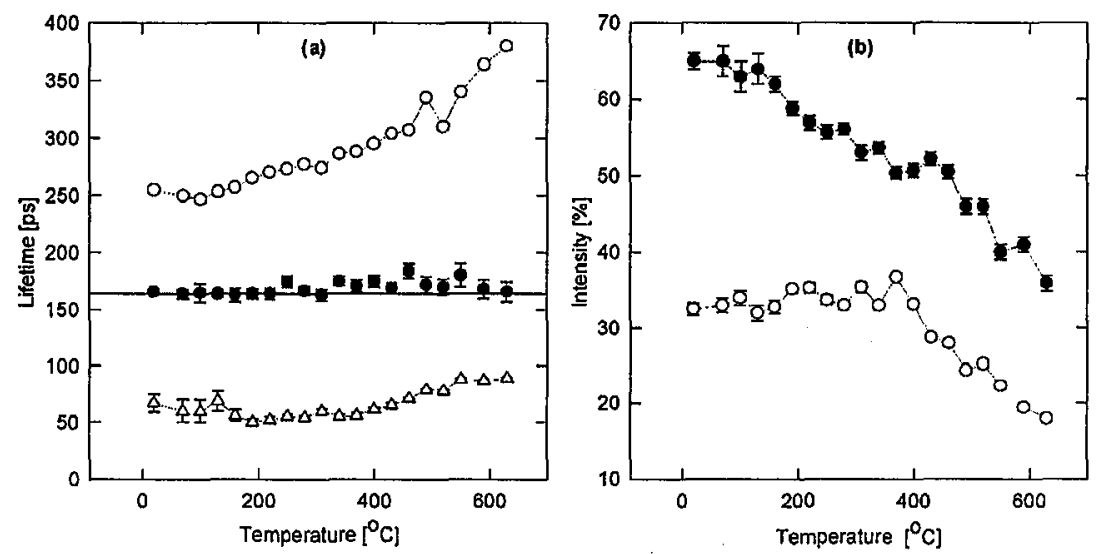

Fig. 1. (a) Lifetimes of individual components as a function of annealing temperature. $-\Delta-$ lifetime $\tau_{1} .-\bullet-$ lifetime $\tau_{2}$ belonging to preliminary fit discussed in Sec. 2 . It testifies temperature independence of positron lifetime at dislocations. In final fit $\tau_{2}$ was fixed at value $164 \mathrm{ps}$ (solid line). - o- lifetime $\tau_{3}$ ("nanovoids"). (b) Temperature dependence of relative intensities of defect components. $-\bullet-I_{2}$ (dislocations), $-0-I_{3}$ ("nanovoids").

Our interpretation is also supported by positron lifetime study of deformed polycrystalline $\mathrm{Cu}$ [13] which revealed presence of dislocation component with almost temperature independent lifetime $160 \mathrm{ps}$ and another component with characieristic lifetime $270 \mathrm{ps}$.

Three-state conventional trapping model (CNM) [11] cannot be applied in interpretation of measured PLS for the following reasons. Usual test of CTM self-consistency consists in checking of validity of equation

$$
\tau_{\mathrm{f}}^{\mathrm{EXP}}=\tau_{\mathrm{f}}^{\mathrm{CTM}}
$$

where

$$
\tau_{\mathrm{f}}^{\mathrm{EXP}}=\left(\sum_{i=1}^{3} I_{i} / \tau_{i}\right)^{-1}, \quad \tau_{\mathrm{f}}^{\mathrm{CTM}}=\tau_{\mathrm{B}} .
$$

For our case we obtain

$$
\tau_{\mathrm{f}}^{\mathrm{EXP}}=176.9 \pm 0.3[\mathrm{ps}]
$$

while

$$
\tau_{\mathrm{f}}^{\mathrm{CTM}}=114.5 \pm 0.1[\mathrm{ps}] .
$$

Clearly Eq. (1) is not satisfied which is direct indication that the assumptions of CTM are not fulfilled.

It is necessary to take into account the existence of two completely different environments in NC materials: defect-free regions inside grains and distorted regions along GB's. Therefore a modified trapping model (M'TM) was developed in the present work. The new symbols used in the model are listed below.

$\lambda_{\mathrm{B}}=1 / \tau_{\mathrm{B}}-$ annihilation rate of free positrons.

$\lambda_{\mathrm{D}}=1 / \tau_{\mathrm{D}}-$ annihilation rate of positrons at dislocations. 
$\lambda_{\mathrm{N}}=1 / \tau_{\mathrm{N}}-$ annihilation rate of positrons in "nanovoids" ( $\tau_{\mathrm{N}}$ is positron lifetime in "nanovoids").

$n_{\mathrm{B}}(t)$ - probability that positron is alive as free at time $t$.

$n_{\mathrm{D}}(t), n_{\mathrm{N}}(t)$ - probability that positron is trapped at time $t$ by a dislocation or "nanovoid", respectively.

$K_{\mathrm{D}}, K_{\mathrm{N}}$ - trapping rate at a dislocation and "nanovoid", respectively, for positron situated inside distorted region.

$\eta$ - volume fraction of distorted regions.

Assumptions of MTM can be summarized as:

1. Positrons annihilate in the non-distorted regions from free state only, however, inside distorted regions all positrons annihilate from trapped state in dislocation or "nanovoid".

2. Positrons can move among distorted and non-distorted regions by diffusion. Probability that free positron will be found in a distorted region equals $\eta$.

3. At time $t=0$ after thermalization positron in non-distorted region is free but positron in distorted region is trapped by dislocation or "nanovoid".

4. No detrapping occurs.

With respect to these assumptions, MTM kinetic equations get the form

$$
\begin{aligned}
& \frac{\mathrm{d} n_{\mathrm{B}}}{\mathrm{d} t}=-(1-\eta) \lambda_{\mathrm{B}} n_{\mathrm{B}}-\eta\left(K_{\mathrm{D}}+K_{\mathrm{N}}\right) n_{\mathrm{B}}, \\
& \frac{\mathrm{d} n_{\mathrm{D}}}{\mathrm{d} t}=-\lambda_{\mathrm{D}} n_{\mathrm{D}}+\eta K_{\mathrm{D}} n_{\mathrm{B}} \\
& \frac{\mathrm{d} n_{\mathrm{N}}}{\mathrm{d} t}=-\lambda_{\mathrm{N}} n_{\mathrm{N}}+\eta K_{\mathrm{N}} n_{\mathrm{B}} .
\end{aligned}
$$

Boundary conditions can be written as

$$
\begin{aligned}
& n_{\mathrm{B}}(t=0)=1-\eta, \\
& n_{\mathrm{D}}(t=0)=\eta \frac{K_{\mathrm{D}}}{K_{\mathrm{D}}+K_{\mathrm{N}}}, \\
& n_{\mathrm{N}}(t=0)=\eta \frac{K_{\mathrm{N}}}{K_{\mathrm{D}}+K_{\mathrm{N}}} .
\end{aligned}
$$

PLS shape $\mathcal{S}(t)$ is directly connected with the solution of the kinetic equations by

$$
\mathcal{S}(t)=\frac{-\mathrm{d}\left(n_{\mathrm{B}}+n_{\mathrm{D}}+n_{\mathrm{N}}\right)}{\mathrm{d} t}=\sum_{i=1}^{3} \lambda_{i} I_{i} \exp \left(-\lambda_{i} t\right) .
$$

Inserting the solution of Eqs. (3) to Eq. (5) gives for annihilation rates $\lambda_{i}$ $\left(\lambda_{i}=1 / \tau_{i}\right)$ and relative intensities $I_{i}$ of the individual spectral components

$$
\lambda_{1}=(1-\eta) \lambda_{\mathrm{B}}+\eta\left(K_{\mathrm{D}}+K_{\mathrm{N}}\right), \quad \lambda_{2}=\lambda_{\mathrm{D}}, \quad \lambda_{3}=\lambda_{\mathrm{N}}
$$




$$
\begin{aligned}
& I_{1}=1-I_{2}-I_{3} \\
& I_{2}=\eta K_{\mathrm{D}}\left(\frac{1}{K_{\mathrm{D}}+K_{\mathrm{N}}}+\frac{1-\eta}{\lambda_{1}-\lambda_{\mathrm{D}}}\right), \\
& I_{3}=\eta K_{\mathrm{N}}\left(\frac{1}{K_{\mathrm{D}}+K_{\mathrm{N}}}+\frac{1-\eta}{\lambda_{1}-\lambda_{\mathrm{N}}}\right) .
\end{aligned}
$$

Set of non-linear Eqs. (6) was numerically resolved for as-prepared state of $\mathrm{n}-\mathrm{Cu}$ with experimental values $\lambda_{i}, I_{i}(i=1,2,3)$ (Table) and $\lambda_{\mathrm{B}}$. The following solution was obtained:

$$
\begin{aligned}
& \eta=33.2[\%], \\
& K_{\mathrm{D}}=1.74 \times 10^{10}\left[\mathrm{~s}^{-1}\right], \\
& K_{\mathrm{N}}=1.00 \times 10^{10}\left[\mathrm{~s}^{-1}\right] .
\end{aligned}
$$

Self-consistency of MTM application can be verified using relation similar to Eq. (1)

$$
\tau_{\mathrm{f}}^{\mathrm{EXP}}=\tau_{\mathrm{f}}^{\mathrm{MTM}}
$$

where

$$
\tau_{\mathrm{f}}^{\mathrm{MTM}}=\left[(1-\eta)^{2} \lambda_{\mathrm{B}}+\eta \frac{K_{\mathrm{D}} \lambda_{\mathrm{D}}+K_{\mathrm{N}} \lambda_{\mathrm{N}}}{K_{\mathrm{D}}+K_{\mathrm{N}}}\right]^{-1} .
$$

For as-prepared state of n-Cu Eq. (8) gives

$$
\tau_{\mathrm{f}}^{\mathrm{MTM}}=176.8[\mathrm{ps}],
$$

which is in very good agreement with $\tau_{\mathrm{f}}^{\mathrm{EXP}}=176.9 \pm 0.3 \mathrm{ps}$.

Trapping coefficients $K_{\mathrm{D}}$ and $K_{\mathrm{N}}$ are directly correlated with dislocation density and "nanovoid" concentration, respectively. Dislocation density in non-distorted regions is considerably smaller than that in distorted regions. Hence, mean dislocation density can be expressed as

$$
\varrho_{\mathrm{D}}=\eta K_{\mathrm{D}} / \nu_{\mathrm{D}}
$$

where specific positron trapping rate for $\mathrm{Cu}$ dislocations $\nu_{\mathrm{D}}$ can be estimated by comparison with $\varrho_{\mathrm{D}}=1 \times 10^{14} \mathrm{~m}^{-2}$ measured by XRD on the same state of the material [7]. The estimation is

$$
\nu_{\mathrm{D}} \approx 0.6 \times 10^{-4}\left[\mathrm{~m}^{2} \mathrm{~s}^{-1}\right] .
$$

All "nanovoids" are situated inside distorted regions, therefore, one can write for their mean concentration

$$
c_{\mathrm{N}}=\eta K_{\mathrm{N}} / \nu_{\mathrm{N}} .
$$

On the assumption that free volume of a "nanovoid" is about 5 vacancies, we can approximate specific positron trapping rate $\nu_{\mathrm{N}}$ for "nanovoids" by $\nu_{\mathrm{N}} \sim 5 \nu_{1} \mathrm{~V}$, where $\nu_{1 \mathrm{~V}}=(1.2 \pm 0.2) \times 10^{14} \mathrm{~s}^{-1}$ at. is specific positron trapping rate for $\mathrm{Cu}$ monovacancy [11]. Thus the estimated mean concentration of "nanovoids" is

$$
c_{\mathrm{N}} \approx 6 \times 10^{-6} \text { [at. }^{-1} \text { ]. }
$$




\subsection{Isochronal annealing of $n-C u$}

Lifetimes and relative intensities of individual components as a function of annealing temperature are shown in Figs. 1a and 1b, respectively. Application of MTM gives information about behaviour of volume fraction of distorted regions $\eta$ with increasing temperature, see Fig. 2a. The estimation of temperature dependence of mean dislocation density $\varrho_{\mathrm{D}}$ and "nanovoid" concentration $c_{\mathrm{N}}$ obtained using Eqs. (9) and (10), respectively, is shown in Fig. 2b. In all cases the application of MTM is fully justified by satisfying Eq. (7). It should be pointed out that the estimation of $c_{N}$ is only a poor approximation because the increase in $\tau_{3}$ with increasing temperature (Fig. 1a) is not taken into account.
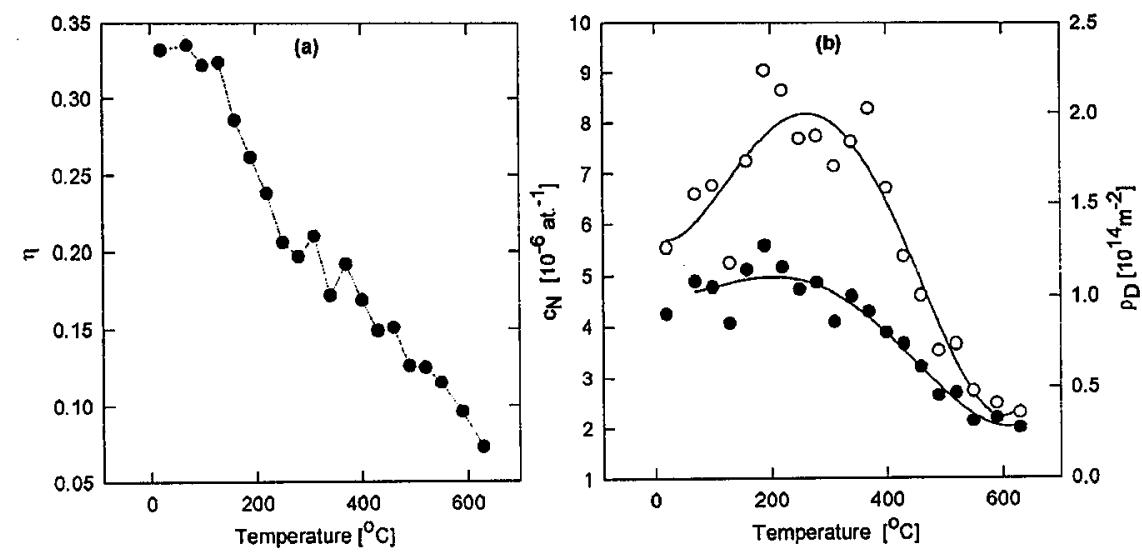

Fig. 2. (a) Dependence of volume fraction of distorted regions $\eta$ on annealing temperature. (b) - - mean dislocation density $\varrho_{D},-0-$ mean concentration of "nanovoids" $c_{N}$ both estimated by MTM as a function of annealing temperature. Solid lines serve only to guide eyes.

Figure 1a shows the increase in lifetime of "nanovoids" with increasing annealing temperature. This effect may be caused by change of non-equilibrium GB's towards equilibrium GB's which is connected with relaxation of elastic stresses. Such changes were observed by TEM in various NC materials prepared .by SPD, e.g. in [5].

Several stages can roughly be recognized in annealing curves of relative intensities (Fig. 1b).

- Below $130^{\circ} \mathrm{C}$ no changes occur.

- In the temperature range $130-280^{\circ} \mathrm{C}, I_{2}$ exhibits substantial decrease and there seems to be a little increase in $I_{3}$. However, mean dislocation density $\varrho_{D}$ remains constant as one can see from Fig. $2 \mathrm{~b}$. Therefore, the decrease in $I_{2}$ is probably due to rearrangement of dislocations and narrowing of distorted regions. The latter is clearly indicated by decrease in $\eta$ (Fig. 2a). Smooth increase in $I_{3}$, if any, is rather surprising but one can see the same effect also on $c_{\mathrm{N}}$ (Fig. 2b). It may appear as a consequence of reducing dislocation shielding around "nanovoids" due to narrowing of distorted regions. This means that actual value of mean concentra- 
tion of "nanovoids" in the material should be estimated in the temperature interval $190-370^{\circ} \mathrm{C}$ where $c_{\mathrm{N}}$ reaches its maximum and remains constant. Application of the same procedure as in the previous section and averaging over temperature range $190-370^{\circ} \mathrm{C}$ gives

$c_{\mathrm{N}} \approx 8 \times 10^{-6}$ [at. $^{-1}$ ].

Note that the similar increase in the component with lifetime of about 270 ps was also observed during isochronal annealing of deformed $\mathrm{Cu}$ [13].

- Above $280^{\circ} \mathrm{C}$, decrease in $\varrho_{D}$ begins (Fig. $2 \mathrm{~b}$ ) and is accompanied with decrease in $I_{2}$ (Fig. 1b). On the other hand, the mean concentration of "nanovoids" remains approximately constant in the temperature range $280-370^{\circ} \mathrm{C}$ (Fig. $2 \mathrm{~b}$ ). Thus no grain growth occurs below $370^{\circ} \mathrm{C}$.

- At temperature $370^{\circ} \mathrm{C}$, rapid decrease in $I_{3}$ starts. It is direct indication of grain growth onset which is supported also by observed decrease in $c_{\mathrm{N}}$ (Fig. 2b).

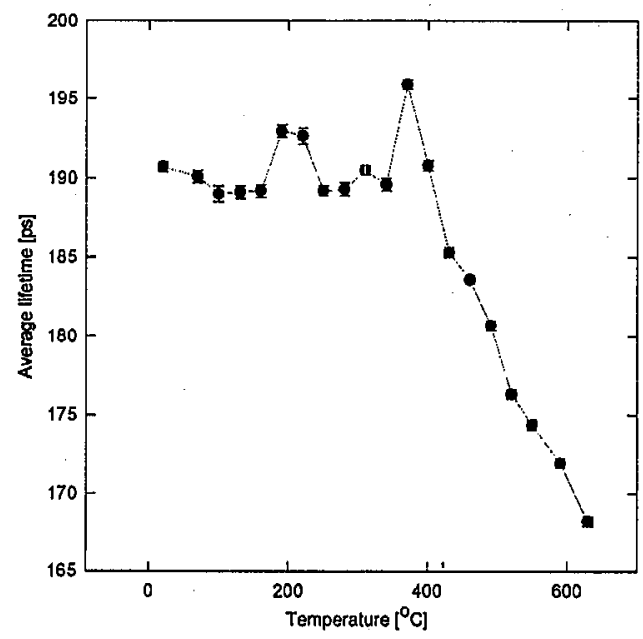

Fig. 3. Mean lifetime dependence on annealing temperature.

Mean lifetime shown in Fig. 3 is approximately constant up to the onset of grain growth $\left(370^{\circ} \mathrm{C}\right)$. Then this quantity decreases with increasing annealing temperature. From Fig. $1 b$ one can realize the decrease in $I_{2} / I_{3}$ ratio at temperatures region below $370^{\circ} \mathrm{C}$, where $I_{2}$ decreases but "nanovoids" are not being annealed out yet. Nevertheless, above $370^{\circ} \mathrm{C}$ grain growth occurs and "nanovoids" recover more rapidly than dislocations. This is reflected by a positive slope of temperature dependence of the $I_{2} / I_{3}$ ratio.

\section{Conclusion}

1. Positrons are trapped by dislocations or "nanovoids" in distorted regions of n-Cu prepared by SPD. Highly non-uniform defect distribution does not allow the application of CTM.

2. Considerations consequentially based on NC structure lead to development of MTM. Behaviour of positrons in n-Cu prepared by SPD can be self-consistently interpreted in terms of this model. 
3. Rearrangement of dislocations accompanied with decrease in mean dislocation density with increasing temperature precedes grain growth. The onset of grain growth can be clearly recognized at $370^{\circ} \mathrm{C}$ due to decrease in mean concentration of "nanovoids".

\section{References}

[1] R.Z. Valiev, E.V. Kozlov, Yu.F. Ivanov, J. Lian, A.A. Nazarov, B. Baudelet, Acta Metall. Mater. 42, 2467 (1994).

[2] R. Birringer, Mater. Sci. Eng. A 117, 33 (1989).

[3] O. Schneeweiss, J. Phys. Condens. Matter 1, 4749 (1989).

[4] R.Z. Valiev, Mater. Sci. Eng. A 234-236, 59 (1997).

[5] R.Z. Valiev, A.V. Korznikov, R.R. Mulyukov, Mater. Sci. Eng. A 168, 141 (1993).

[6] I.V. Alexandrov, K. Zhang, A.R. Kilmametov, K. Lu, R.Z. Valiev, Mater. Sci. Eng. A 234-236, 331 (1997).

[7] R.K. Islamgaliev, F. Chmelík, R. Kužel, Mater. Sci. Eng. A 237, 43 (1997).

[8] F. Bečváŕ, L. Lešták, I. Novotný, I. Procházka, F. Sebesta, J. Vrzal, Mater. Sci. Forum 175-178, 947 (1995).

[9] F. Bečváŕ, J. Ćǐžek, I. Procházka, Acta Phys. Pol. A 95, 448 (1999).

[10] I. Procházka, I. Novotný, F. Bečváŕ, Mater. Sci. Forum 255-257, 772 (1997).

[11] P. Hautojärvi, F. Corbel, in: Proc. Int. School of Physics "Enrico Fermi", Course CXXV, Eds. A. Dupasquier, A.P. Mills Jr., IOS press, Amsterdam 1995, p. 143.

[12] H.-E. Schaefer, W. Stuck, W. Bauer, F. Banhart, Mater. Sci. Forum 15-18, 117 (1987).

[13] A.P. de Lima, C. Lopes Gil, D.R. Martins, N. Ayres de Campos, L.F. Menezes, J.V. Fernandes, in: Proc. European Meeting on Positron Studies of Defects, Vol. 2, Part 1, Eds. G. Dlubek, O. Brümmer, G. Brauer, K. Hennig, Martin-Luther-Universität Halle-Wittenberg, Wernigerode 1987, p. C1.

[14] B.T.A. McKee, S. Saimoto, A.T. Stewart, M.J. Scott, Can. J. Phys. 52, 759 (1974).

[15] A. van den Beukel, in: Proc. Int. Conf. Vacancies and Interstitials in Metals, Eds. A. Seeger, D. Schumacher, W. Schilling, J. Diehl, North-Holland, Amsterdam 1970, p. 427.

[16] I. Kanazawa, H. Murakami, M. Doyama, Phys. Status Solidi A 103, 403 (1987).

[17] S. Mantl, W. Trifthäuser, Phys. Rev. B 17, 1645 (1978).

[18] M.J. Puska, R.M. Nieminen, J. Phys. F 13, 333 (1983).

[19] M. Eldrup, P.G. Sanders, J.R. Weertman, Mater. Sci. Forum 255-257, 436 (1997). 